\title{
Vocal Ecosystems Beyond Pedagogy: Working With Challenges
}

\author{
Jeri Brown \\ Concordia University \\ Canada
}

\begin{abstract}
Knowledge of vocal improvised music, whether demonstrated by high or low obscure pitch sounds, the beating of the chest while making music sounds, vocal pitch matching or vocal animation with or without the use of technology, has paved the way for a steady stream of vocal artists through the years, each dedicated to vocal exploration. While jazz vocal improvisation appears on the surface to involve few or no rules, it is a form of communication between artist and listener, where the artist adheres to a set of rules or principles. Here the jazz improviser is treated as part of an ecosystem, a concept in the biological sciences that comprises a set of interacting organisms and environments in a particular place. Within the jazz vocal improvisational ecosystem there are various roles, approaches and activities. What follows is a description showing how the analogy with biological ecosystems can be applied to a 'vocal ecology', how this viewpoint may also aid the improvising jazz. vocalist in artistic expression and considerations for the vocalist with vocal difficulties in communication.
\end{abstract}

\section{Introduction}

The singing voice is evidenced primarily through a combination of sonancy, intonation and cadence. Such evidence informs the listener and singer of the quality of vocal production and provides every singer with a unique fingerprint of sound. In jazz, the execution of vocal delivery is subjective largely due to the taste of the listener and the preparedness of the singer. Acquiring a trained skill level through dedication, instruction and regular practice remains a crucial element for attaining and improving vocal performance credibility.

In the co-written composition of Kenny Wheeler's Gentle Piece with Jeri Brown lyric, a high level of vocal credibility is demonstrated through the use of music intelligence as a central part of the communication process between voice and flugelhorn. The use of tone matching further enhances the harmonic musical language to reinforce a mood between voice and flugelhorn along with jazz piano, bass, and drums. Tone matching is a characteristic of vocal language. In Who Are You, another Kenny Wheeler composition, a high level of vocal credibility is also demonstrated in the communication of the two principal artists. The use of tone matching also stimulates the harmonic musical language and mood in this piece. In both pieces, composer, trumpet and flugelhorn player Kenny Wheeler creates poetic artistry with voice, tenor and arranged orchestra. Horn and voice are well suited to the complex material fusing personas in the signature over layering of cadenzas that reoccur in his works with a focus on emotion and sentiment [4].

The use of vocal language and musical intelligence in improvisation creates added stimulation in the improvised vocal delivery.

\section{Characteristics of jazz vocal improvisation}

The jazz singer uses their senses to work with air, heart, soul, throat, diaphragm, tongue, guts and various other organs necessary to produce vocal experimental wonders. Key characteristics necessary for the jazz vocal improviser include:

.Personalized sound

.Demonstrated ability to explore and integrate discovery in the actualization, consumption and delivery of music, sound, noise and silence

.Ability to work with others in the creative processing of improvised music

.Internal feeling of chord roots

.Refined working knowledge of musical intelligence as in song structure, melody, harmony, and rhythm

.Instrumental awareness

.Animated, creative, whimsical temperament Vocal technique characteristics of the jazz vocal improviser include:

.Fluid tonal delivery throughout registers

.Pristine, smooth vocal expression

.Clear, limber ornamentations

.Proficient breath support

.Use of performative inflections when stating phrases

.Perspicuous diction in the use of words as in lyrics, song stories, nonsense syllables and sub-languages 


\section{The improviser as part of an ecosystem}

In the biological sciences an ecosystem is a concept that comprises a set of interacting organisms and environments in a particular place. Each system has its own set of musicians, styles and interactions. These systems overlap, merge into one another and are probably impossible to circumscribe perfectly. Hip-hop, avant-garde, jazz, swing and standards are all examples of music systems each with its own styles, performers, and rules.

Similarly, a vocal ecosystem is a concept that provides a systematic approach which may be useful during the process of vocal improvisation. The concept comprises a set of interacting domains that enhance musical stimuli in a particular way. These domains behave like living organisms because they evolve, propagate their organization and interact among themselves. When utilized in conscious free play they form the basis of a type of communication between artist and listener [2].

When jazz vocalizing involves a deliberate quest for improvisation, the singing performer may intentionally use stimuli to prompt imitative behavior. The implementation of the four principal domainsvocal language, culture, sound and environment enhances musical stimulation in vocal improvisation. For the jazz vocalist, including one or more of these domains in a musical improvisation will aid her ability to create, build and participate in improvisation.

The creation of vocal ecosystem as a term borrows parts of its philosophy from the 1969 study by anthropologist Ralph Holloway of contrasting human and non-human primate learning strategies. He describes the "ratchet effect" as a method that has enabled humans to evolve and adapt to complex social systems. In 1999 comparative psychologist Michael Tomasello expanded the metaphor to shed light on the evolution of human culture by renaming it the "cultural rachet". Adding, "Once a certain invention has been made, it can jump from one mind to another (by means of imitation) and thus a whole population can acquire a new trait..." [7].

A similar effect takes place in the interaction of the jazz vocal improviser between the contributing musicians and listener. When one vocally transmitted musical idea, as in a phrase, triggers a significant reaction from the musicians with continuing or repetitive effect in some form, during the improvised work, it is as if a main idea has been adopted as a new trait. Seeking such stimulation becomes a key factor in aiding the creative and musical interaction of the vocalist.

\section{Vocal language domain as improvisation aid}

In the vocal language domain the vocal improviser uses music as a language to communicate and create a body of music, often using music as a metaphor from which to resonate an authentic texture of heartfelt personal expression. Vocalists may also use their voice as an instrument to further break apart limits of traditional communication, thus pioneering sounds with and without the use of effects that transmit language to convey meaning. Much like systems in sciences, a vocal improviser equipped with a common language system can be stimulated to communicate.

In Jimmy Rowles' The Peacocks, the use of an invented vocal language and ethereal environment domain aids the vocal improviser in painting a story about two peacocks while interacting with a Chinese flutist with jazz piano, bass and drum. With Michel Dubeau on flute, sensuality is enhanced in the call and response of the voice and shakuhachi flute, relating to the senses of sight, smell, hearing and touch [9]. The vocalist's expressive powers are heard at their best on this set during her lengthy, wordless interplay with flute. We hear the improvising voice and Chinese flute interacting in a seemingly seamless erotic communication where the sounds of the two 'voices' begin to connect with the listener as if they were both of human form in conversation [4]. Here the vocal improviser deconstructs American jazz pianist and composer Jimmy Rowles' classic turning it into a rich vocalization.

In a later recording of The Peacocks aka A Timeless Place with Rowles at the piano, this classic 1994 lyrical vocalese features the same vocal improviser with added lyrics by Norma Winstone without disturbing the original ethereal environment domain aid [8].

Joint vocal engagement or joint vocal attention describes a process where the vocal improviser recognizes herself as an intentional agent in the ensemble. Her ability to demonstrate musical leadership, skill and power allows the singer to concurrently select and demonstrate vocal choices leading the musicians toward a type of communal cohesiveness.

In the initial recording of The Peacocks musical leadership is exhibited through the vocal improviser's choices of sound and vocal texture. It stimulates interaction between voice, flute, bass and piano demonstrating a joint engagement with all musical participants committed to enhancing and supporting the vocal improvisation. The delicate, sensual improvisation is handed down by the vocal improviser's light, clear-tone delivery setting the tone for Rufus Reid on bass and Kirk Lightsey on piano to follow in kind with key aesthetics. 
In Brown's Time Suite (aka 10.20) the use of inventive vocal language, sound technology and ethereal environment domains aid the vocal improviser when painting a story about the passage of time while interacting with jazz pianist Fred Hersch in a wordless improvisation. Leon Thomas' Echoes features use of vocal language and culture domains as a device for storytelling the modal, Coltrane-ish piece by vocal improvisers Jeri Brown and Leon Thomas as they trade yodels, yelps, oohs and ahhhs with jazz piano, bass, and drums [9].

Jeri Brown \& Sandy Moore's Chasing Cain and Mariner's Mourn (both compositions for film), feature vocal language and sound technology domains as devices for the vocal improviser to set a mood while interacting with world music ensemble and string quartet. The overlaying of voices provides a unique blending of cultural sources with resonating texture. The effect of Moore's use of the music as a metaphor expresses an authentic texture of personal expression for the jazz vocal improviser's classic and experimentalist delivery [8]. In Chasing Cain, Moore's treatment for film stems from an originally improvised vocal melody is empirically shaped from a short composition with improvised vocal development to that of a final cut for a creative and impressionable work.

\section{Culture domain}

Vocal ecosystem compositions that reflect a unique blending of cultural sources (e.g., Caribbean, eastern European, spiritual), add personal vocal artistry and ultimately increase the expression of an emotionally communicative style often depicting ethno-chanting, ethno-rhythms and ethno-dance. In Harold Faustin's Tam Tam Dance, the combined use of culture, sound technology and vocal language domains aid the vocal improviser while interacting with Harold on guitar and world music ensemble. In this example linguistic nonsense syllables are used as a vehicle to stimulate musical expression. Voice and guitar interact in sync throughout the Haitian jazz folkloric piece in a danceable fashion. Prerecorded vocal sounds are layered, stacked and overdubbed atop each voice of the sole vocal improviser as directed by the composer. Great technique and innate sense of adventure take this musical journey into new territory where classical, jazz and Afro-West Indian rhythms become one. This form of vocal improvisation instruction most readily occurs in the recording studio process and requires the vocalist to bring in all of their 'chops' as well as music literacy.

Brown's Echo Thoughts, and African Echo display enhanced improvisation with the inclusion of culture and invented vocal language domains to aid the vocal improviser in an emotional interaction between master drummer Clayton Cameron and voice.
In Avery Sharpe's Uncle from Ghana the use of culture domain is used through the combination of rhythm and modal music to aid the vocal improviser in a soulful interaction between voice and master acoustic bassist Avery Sharpe.

\section{Sound domain}

The use synthetic and manufactured, looped or layered technical sound is created in studio or on location with appropriate equipment. Brown's four compositions for dance: Arising, Mid Day, Celestial Flight, and Night utilize sound technology design domain to aid vocal improvisation between voice, jazz pianist and accordionist. Improvisation is further enhanced through the expertise of sound design engineer Mark Corwin.

Sound domain is often combined with an additional domain to place it in a context. In the final domain example Soul Shower owes its aids to a combination of vocal language, sound technology and environment domains.

\section{Environment domain}

In Jeri Browns' composition Soul Shower, spoken and vocalized overlays utilize inventive vocal language, sound technology and environment domains to aid the vocal improviser in poetic expression while interacting with multiple voices, and soulful rhythms combining jazz piano, bass, and drums. With the support of sound engineer Dennis Moody, words, phrases and sentences are sequenced and used as a vehicle to create a musical work that tells a story.

\section{Quest for vocal corrections}

A jazz vocalist draws upon sound a great deal during performance. Subtle changes in color and timbre in the voice can evoke different emotions during the delivery of a song. In the process of improvising on sound the singer becomes more familiar with an ability to alter and enhance sounds that are personal and unique.

The better the tone, the better the message is communicated. In the vocal language domain the vocal improviser uses music as a language to communicate and create a body of music, often using music as a metaphor from which to resonate an authentic texture of heartfelt personal expression. Vocalists may also use their voice as an instrument to further break apart limits of traditional communication, thus pioneering sounds with and without the use of effects that transmit language to convey meaning. Much like systems in sciences, a vocal improviser equipped with a common language system can be stimulated to communicate. 
With an ever-increasing need to instruct students with special needs, teachers are challenged to consider and attend to a variety of issues.

When the singer has problems communicating, a further investigation by singer, teacher, and possible medical professional might be necessary to establish clarity and to work through a vocal study to improve the vocal delivery. In such cases a phonatory evaluation may be helpful.

One communication impediment could be neurological. Neurologic voice disorders can be isolated problems or occur in the context of extensive systemic disease.

Vocal obstructions may also hinder the tonal quality of the singers and impede communication. These include disorders for which voice problems and complaints may initially present to the laryngologist or voice clinician; and disorders for which medical, surgical, or behavioral intervention may improve laryngeal function, in the context of the individual's general physical condition.

Vocal rest or practice in changing the pitch centers in favor of a lesser-used part of the vocal range may be recommended as one method of developing tonal strength.

Resonance practice is another aid to help to identify and adjust timbre and pitch in the voice to help the identification of a preferred vocal sound. In many ways the ability to control the variations in resonance can dramatically affect the vocal performance.

Such considerations should not be minimized. There is no getting around the fact that a singer who wishes to pursue the world of jazz must be obsessed and convinced of the standards of excellence that have been achieved from the masters of this vocal art form. Today pop music and world music also provide a wonderful palette for the jazz vocalist. From the 60's to today, from primitive, ritual songs of various cultures to alternative popular urban music including hip hop influences, jazz vocalizing is not about singing time period pieces, but, rather, it is about conveying a consistent, memorable personal vocal message that conveys a strong sense of musicianship adaptability, versatility and facility of your instrument. The ability to hear clearly, to receive messages and to deliver clear messages in the communication process is necessary for improvisation to occur. Tone is a particular quality of the sound of any voice or instrument. It can have a direct effect on setting a mood.

Ultimately, good vocal technique and awareness of it can help a jazz vocalist to remain true to their sound.

\section{Aids for Improving Tone}

Aids for improving tone include, first, assisting and assigning the singer to practice singing a familiar tune as written. Second, having the singer record herself singing the same tune with specific focus on her sound vocabulary to distinguish a sense of her persona when singing. Third, establishing a journal through this process to increase self-awareness and focus in improving this technique. Fourth. Help the singer to make a short list of sounds that they hear and pay attention to immediately, sounds that draw them in, along with those sounds that they hear, perhaps, everyday, but rarely focus on. Fifth. Help the singer listen for the different shadings and textures of the vocal sounds when singing. Sixth. Record the singer in several songs of their own choice. Seventh. Direct the singer to make note of the color, and integrity of personal sounds in each song as part of the personal vocal inventory. Eighth.

Assist the singer in differing the pitch levels from high or low to increase variety of intense - more relaxed sound frequencies to alter the perception of pitch. Such attention including the slightest wavering (sharp or flat) of pitch can greatly effect the mood and harmony of the vocal improviser to respond to the communication of music. Ninth. Along with individual vocal sound development, assist the singer to make a daily diet of studying and listening to the repertoire of music artists and their songs from the classical period of music with more emphasis on tone.

Applying vocal acoustics of dynamics and duration in singing style provides a rich variety of texture for the improviser. Dynamics refers to degrees of loudness. For good communication, there must be a dynamic blend.

Assist the singer in becoming acquainted with common dynamic markings including: (pp)pianisimo-extremely soft

(p) piano-soft

( $m p)$ mezzo piano-medium soft

(mf) mezzo forte-medium loud

(f) forte-loud

$(<)$ (cresc.) crescendo-increasing power of tone

(>)(descr.) dimenuendo or decrescendogradually diminishing in power of tone

(ff) fortissimo-very loud

Repertoire traditionally used in jazz vocalizing comes from what is known as the jazz idiom. Jazz idiom includes self-penned songs, songs of literature including poetry or prose, and world music. This idiom has typically included songs from films, and stage along with compositions of jazz musicians from the 20's through the 50's. There is much to be gained from studying the contributions of traditional vocal jazz stylists and their repertory as well as that of some current and emerging vocal artists and 
songwriters who have included talent and improvisational instincts in their music making.

Stimulation for the jazz vocalist can come from many sources. From reading a good book to observing or creating a drawing, the idea is for the singer to allow for such an opportunity to feel the character of a person in a fictional setting. This can inspire one to dig deeper when singing creatively. Guide the singer through this process and encourage them to enter thoughts in the journal.

Such reflections often provide inspiration in the creative process. A suggested exercise is to have the singer observe and comment on a series of paintings for emotional relaxation and stimulation. Follow up with these questions for each painting.

1) Is the painting harmonious or disharmonious?

2) Does it make you calm?

3) Does it upset you?

4) Does it leave you feeling nothing?

5) Do you think any parts are unnecessary?

6) Does the creativity that you feel was required to make this art inspire you to be creative in your own art?

When reading something from poetry or something longer, the idea is to allow for an opportunity to feel the character of a person in a fictional setting - digging deeper when singing creatively.

\section{Anatomy of the Jazz Vocalist}

There are so many different ways of describing singers who have signature vocal sounds and who improvise with recognizable, consistent vocal qualities. One singer might sing on the edge of the voice with an aspirated, airy, light vocal quality. Still another might sing on the inside of the voice with a rich, warm resonated vocal quality. While much can be said technically to describe the voice, eventually it is a matter of personal choice. Either you like it or not.

There are so many different ways of describing singers who have signature vocal sounds and who improvise with recognizable, consistent vocal qualities. To assist in refining vocal listening skills, singers should be encouraged to listen to the popular singers of today for comparison. A few suggested examples include: Bryan Adams, Janis Joplin, Sting, Cher, Elvis Presley, Michael MacDonald, Josh Groban, Feist, Amy Whinehouse, Beyonce, Dave Matthews, Sting, and Brian Albright.

Several of pop and world singers display many jazz vocal ingredients in their song delivery. This vocal fusion effect signals the inevitable influences of jazz vocal technique that have consistently resurfaced throughout the evolution of vocal jazz. Popular music and world music also provide a wonderful palette for the jazz vocalist.
From the 60's to today, from primitive, ritual songs of various cultures to alternative popular urban music including hip hop influences, jazz vocalizing is not about singing time period pieces, but, rather, it is about conveying a consistent, memorable personal vocal message in the majority of a singer's repertoire that conveys a strong sense of musicianship adaptability, versatility and facility of the voice as an instrument. Jazz influenced stylists today may take the popular song of their day and reinvent it, keeping key elements of jazz language present in their arrangements while focusing on the meaning and original context of the song.

A definition of the jazz vocalist includes attitude, responsibility and integrity for the intended message in their song. The vocalist is a messenger. As the bearer of a message set to music, the jazz vocalist becomes the advocate of the text. In this context nothing is more important than reaching out and touching someone with spirit. The goal is to transmit a personal message. Throughout jazz vocal history singers have sought to be advocates and projectors of messages reflected in their attitudes about issues. Issues included love, discrimination, hate, suffering, pain, insensitivity, joy, optimism, pessimism, world issues, community affairs, personal matters, health, welfare, space, time and nature.

During the process of singing a body of music the jazz singer serves as a messenger in the song work transmitting hints of past, present and future beliefs, attitudes and emotions that an audience can relate to. The goal is to reach out and integrate, allowing for a homogeneous experience to take over the energy. The Webster dictionary defines a visionary as one who sees visions. Visions are objects of our imagination. The goal for the jazz singer is to take responsibility for having vision about what is presented musically. The challenge of the jazz vocalist is to act as a sleuth, always in search of a deeper meaning in song work, aspiring to develop into a performer whose creativity stems from technique, spontaneity and vision, becoming an advocate for the song. Choosing this path of advocacy will suggest direction in the way that the song is presented at that particular time. Have the singer describe their creative voice in writing for vocal clarification.

The more senses used to acknowledge creative identity, the more comfortable and consistent the emerging style will be when demonstrating it. The singer should continue to refer to this self description as to build upon for their biography, resume, interview and life journey as a creative vocalist. 


\section{Temperament, diet, energy level of the singer}

Interests operate like perpetual machines for the jazz vocalist. When we become interested in something we pay attention to it, and in doing so we become more interested in it. This process can continue until we become so tired that further concentration or dedication is impossible and we need to stop just to breathe and replenish. But almost as quickly as we have become reinforced we are back again grinding away at what we are interested in. Feeling driven sounds dramatic. And why shouldn't it? Singing from the heart brings out the visionary in you. Feeling driven is a healthy way to feel when singing vocal jazz. Practice will either burn you out or inspire you to become driven as well. When you believe in your music, yourself, and know your direction, it is possible to be creative interpreting and trusting your response to the music based on your experience with it. Such trust can breed more trust each time you encounter the same piece of music, as the results will reinforce your confidence in the undertaking. Thus, you never look forward to redo the song the same way. Instead, you trust your judgment of what is effective in your ability to contribute to the inner meaning that you are already derived from that song as you transmit the essence of that message through your music making. This is known as experiencing. It is the ability to channel yourself in the process of song improvisation while memorizing the lyrics and then singing.. Positioning is what the singer brings to the song when they feel the need to say something about the song that is personal.

Good singers have two important qualities: 1) good breathing technique when singing, and 2) the ability to manipulate the voice for the enhancement of their intended vocal delivery. Energy is present when a healthy approach to singing is utilized. Generally, a vocalist uses two resonances: head and chest. For developing deeper breathing system a few suggestions include the following. First, singers might try to imagine the space from the center chest (just below sternum) as the basement in a building and the roof (crown of head) as the head tone. The singer can imagine sound traveling in the body as if on an elevator traveling up and down from the basement to the roof while maintaining an even dynamic - not too loud and not too soft. While adding singing to the exercise, assist the singer in singing a favorite song the first time as if the pressure is coming from behind the eyes... singing as if it feels like the eyes are swelling or as if about to cry. Singing the second time with the imagination of the pressure coming from the front of mouth...as if there is a buzzing feeling from the lips when singing. Even better is to have the singer imagine singing from the muzzle of the mouth keeping mouth in closed position. The goal is to have each word in the song feels as if it is coming from the same place at the front of the mouth. Second, guide the singer to a comfortable low key sustaining an $\boldsymbol{a h}$ or oh on one breath while ascending major and minor intervals within comfortable range, returning back to the $1^{\text {st }}$ note known as the root. They should take a breath and descend to a comfortable low. Once completed, have them modulate to the next higher key, repeating the exercise until reaching a comfortable high note. Then the singer should try reversing the exercise until returning back to the first root. This exercise should be repeated until there is no need to take a breath in between. Continue this process until you feel as if you are singing all of the notes in your head; then continue the exercise as you sing stepwise lower tones each time. Eventually you will feel like all of the notes are in your chest. Third, work with the singer on interval exercises with jazz syllables. Eventually, this approach will assist in the "literacy of scat used in improvisation.

\section{$\begin{array}{lllllllll}1 & 3 & 5 & 7 & 9 & 7 & 5 & 3 & 1\end{array}$ \\ Shah bah doo wee ooh wee doo bee wah $\begin{array}{lllllllll}9 & 7 & 5 & 3 & 1 & 3 & 5 & 7 & 9\end{array}$ \\ Ooh wee doo bee shah bah doo wee ooh}

Fourth, for better skill development, have singers practice from a recording of drones or roots with a funk or bluesy rhythm while working through these exercises. There are several accompaniment tracks available on line to assist in this process. There are also many tools available from the web. Have singers conduct research to acquire this information. Fifth, ensure that the singer feels free to ad lib once they have mastered these suggested techniques. ad lib simply means to play around with the intervals by changing the interval numbers and the roots. Have fun with it. It is all about building flexibility and confidence in the voice when moving from one resonance to the other. This is not all there is to resonators by any means. It is however a beginning. Mastery does not come easily, and is as ever-changing as is the human condition. Sixth, singers should listen to favorite recordings of instrumental jazz tunes while practicing imitating the sound of each instrument including a saxophone, trumpet, bass, and drum. Have them notice the different tones made by each instrument. There is a particular effect that each independent sound adds to the cohesiveness of the musical group. Have them record in their journal how they felt during this exercise with each instrument that was imitated. Help them to identify which sounds seemed to come easiest from the roof or basement resonator. 
Seventh, sften less is more in jazz vocalizing! Have singers practice mixing volume with resonance for a better delivery. As they select a few songs, for each song have them begin in the chest (basement) with a strong volume. As the melody switches or ascends to higher notes bring the volume down - without increasing the volume just because there are higher notes. They should practice these songs until they have mastered this technique. Eighth, in this exercise singers develop ability to demonstrate versatility by selecting and studying three songs. For each song they should begin at low volume, crescendo to medium loud volume and decrescendo to soft volume on each phrase in each song.

These suggestions for vocal study are intended to strengthen vocal capacity, especially when there is a physical, medical or emotional impediment for the performing singer. Temperament, diet, energy level and many other factors may impede the vocal phonation and timbre thus affecting the outcome for the vocal improviser.

The singer should be encouraged to listen regularly to as much classic jazz as possible. As they listen, assist them in analyzing their favorite vocalists, and instrumentalists according to their most memorable sound. In their journals help them to maintain a discography of their listening assignments. Guide them through the process of listening for trademark characteristics of the singer or instrumentalist. The most important goal is to help them discover what it is about the music experience of improvisation that touches and effects them in a meaningful way. Through repeated hearings and sensory experiences to draw from, they will increase their ability to interpret. A jazz vocalist has a unique and personal quality to their sound that beckons the listener to take them seriously. Something within them reaches out in the sound we hear. Strive to find the inner source of your sound identification. This is the first step to building a storehouse of sounds to keep in your mind and have on call, in order to effectively relate to and use when creating music.

Hear the erupting lava of sound from Sarah Vaughan, or that attitudinal edge of Carmen McRae. That sub tonal deliberateness of Betty Carter, the ragged earthy spirit of Nina Simone. It was a part of their individual storehouse of sounds. Those characteristics gave us an aura that preceded them. And we remembered these qualities as their vocal trademarks.

\section{Vocal Jazz Group Stylists}

Following are suggested examples of vocal jazz groups who provide improvisation. These examples are intended to serve as aids for the singer.
The Swingle Singers; the band debuted in 1962 in Paris by Ward Swingle with Anne Germain, Jeanette Baucomont, Jean Cussac, Christiane Legrand and other singers. The band originally started as backup singers for Charles Aznavour, Edith Piaf, Michel Legrand and other French musicians. Until 2011, the group consisted of two sopranos, two altos, two tenors and two basses. The only instruments accompanying the group are the bass and drums.

The Hi Los is a jazz and pop a cappella quartet composed in the 1950s. The members are Gene Puerling, Bob Morse, Don Shelton, Bob Strasen, Franck Allen Howren and Milton R. Chapman. They are well known for their revolution of the vocal ensemble with two tenors, a bass and a lead singer. The name is a reference to their physical as well as vocal differences. They are primarily known for their use of vocalese and improvised harmonies.

Lambert, Hendricks, and Ross is a jazz vocalese trio eponymous to its members Dave, Jon and Annie, respectively, formed in the 1950s in New York City. They are known for their revolutionary approach to the technique of vocalese, by virtue of their use of big band arrangements (as opposed to the usual intimate, small combos usually associated with vocalese before), as well as their proficiency in improvised harmonies. Annie Ross who had been called upon as a vocalist to train a twelve men choir was later asked to join in the newer initiative of studio multitracking to recreate the arrangement, and so the band was born.

Jon Hendricks; a member of the group mentioned above originally was an $\mathrm{R} \& \mathrm{~B}$ solo singer and lyricist whom enlisted the help of Dave Lambert to record the music to his lyrics for the Woody Herman's "Four Brothers"; from then on, the two formed a team.

Naturally Seven; Originally started by Roger, his brother Warren and five other singers in 1999, in New York City. This group is known for a blend of a cappella and traditional jazz performance, with a passion for distinctive harmonic arrangements. New York Group (New York Voices); a quintet composed of Darmon Meader, Peter Eldridge, Kim Nazarian, Caprice Fox, and Sara Krieger, the band can be said to have formed under the tutelary of Dave, Jazz director at Ithaca College through an alumni band created for the purpose of the European Jazz Festival in the summer of 1986. This group is well renowned for their tight harmonies and a vocal blend, making them comfortable in any setting, from small ensemble to an orchestral arrangement.

The Real Group; is a vocal group based in Sweden. All the members graduated from the Royal Academy of Music in 1989 (Stockholm), versatile in the genres of jazz, pop and Nordic European choir music. This band is well known for the precision of 
their sound and the use of voice as their only instrument.

Take Six; Composed of Claude McKnight, Mark Kibble, Joel Kibble, Dave Thomas, Alvin Chea and Khristian Dentley, formed in Huntsville, Alabama. This band is known for its singers' a cappella arrangements mixed with syncopated rhythms and tight harmonies.

Manhattan Transfer; This a cappella group was created in the Fall of 1972, by Tim Hauser, creator of an earlier Manhattan Transfer combo an aspiring young singer named Laurel Massé. Later on, Hausser met Janis Siegel; although already in a group, Siegel agreed to collaborate on some demos, until she permanently joined the group. Finally, Alan Paul, who was co-starring in the original production of Grease, joined the band. Later on, Janis Siegel (after an accident) did not re-integrate the group and was replaced by Cheryl Bentyne. Andrew Sisters; a band formed in the 1940s from Alabama. These are three sisters led by the youngest, and energetic Patty, followed by Maxene with a harmonic range giving the impression of four rather than three voices, completed the eldest, LaVerne.

All the groups mentioned above hold a common use of the technique of vocalese, with a significant capacity to hold tight harmonies and vary their sound, regardless of the number of singers in the band. They are pioneers of and highly influenced by modern jazz.

\section{Instrumental Jazz Stylists}

I recommend the following suggested examples of instrumental jazz groups of improvisation inspiration. These pivotal artists contributed to the establishment of new styles of a free form of jazz developed in the 1950s and 1960s.

Gil Evans is a Canadian pianist and composer who worked extensively with Miles Davis. Miles Davis is an American trumpet player and bandleader. One of his main associations was with John Coltrane in his John Coltrane is an American saxophone player and composer.

Jon Hendricks is an American singer and lyricist. Other than being of the best scat singers he is also known for writing lyrics on top of very intricate melodies and solos.

\section{Summary}

One of the greatest compliments that I received in my career in print was from a journalist who described my voice. "Her voice can be described as three-dimensional. To the up and down she adds in and out, a plasticity of dynamics stretched and deepened by changes of vowel and tonal color, from breathy contralto to high, flute-like tones." I thought wow, he got me! He hears what I feel and try to convey in my musical expression.

It is not always the case that all others can receive what you attempt to communicate. A singer becomes a stylist, acquiring vocal skill and discipline for interpretation. A singer can not fully appreciate their own sound as others do, so we must try to send clear messages musically with the hope that we can be understood. A jazz singer tells a story and conveys the nuances of a personal philosophy and a particular sound. A jazz vocalist has a personal sound that remains virtually unchanged over time even if vocal range, versatility and vocal technique are improved or distorted. It is where the presence of color and texture is well established and present in the voice, out front in their song delivery. Like a banner, the singer wears it in vocal delivery identity. It is that aura that precedes the literal singer.

Vocal ecosystem is an aid to the jazz vocalist reaching for new adventures and frontiers when improvising. It is offered as a concept to encourage the self-directed collaboration of vocalists, artists and technologists in vocal improvisational performance.

The vocal improviser's performance can be aided by incorporating as many domains as possible in an inclusive and participatory manner with fellow musicians. Such benefit can stem from planning an improvisational strategy before engaging in the activity that includes a systematic approach to enhance the production, consumption and performance of a song creation. As part of vocal ecology vocal ecosystem offers a supportive stimulating tool for enhancing creativity in vocal performance. When jazz vocalizing involves a deliberate quest for improvisation, the singing performer may intentionally use stimuli to prompt imitative behavior. The interaction of the four principle domains: vocal language, culture, sound and environment are offered to enhance musical stimulation in vocal improvisation, furthering jazz vocal improvisation exploration. For the jazz vocalist, including one or more of these domains in a musical improvisation will aid her ability to create, build and participate in improvisation. For further related information see also [1], [2], [3], [5] and [6].

\section{References}

[1] R. Tallis, Aping Mankind, Routledge, London and New York, 2014.

[2] S. Nachmanovitch, Free Play: Improvisation in Life and Art, Penguin Group, 1990.

[3] D. Liebman, Self-portrait of a jazz artist: Musical thoughts and realities, Advance, 1988. 
[4] J. Brown, Creating Improvisation - Voice in Action through Vocal Ecosystems, Jongleur Press, 2013.

[5] V. L. Wooten, The Music Lesson: A Spiritual Search for Growth Through Music, 2008.

[6] C. Lane, You Can Sing If You Want To, Simon \& Schuster, 1994.

[7] Google reference listings:

http://en.wikipedia.org/wiki/Singing

http://en.wikipedia.org/wiki/Ratchet_effect http://

cobussen.com/research/auditory-culture http://

en.wikipedia.org/wiki/Joint_attention

[8] The Penguin Guide to Jazz on CD, 7th Edition, Penguin, 2004.

[9] All Music Guide, 2nd Edition, Miller Freeman Books, 1996.

\section{Acknowledgements}

I would like to thank M. Johnston, M. Charland, and B. Freedman for discussion; M. Corwin, and D. Moody for sound technology design; and D. Trible, M. David, C. Cameron, F. Hersch, A. Sharpe, H. Faustin, S. Moore, M. Dubeau, S. Sloutsker and S. Popa for musical contributions. 